\title{
Audible Noise Measurement and Analysis of the Main Power Apparatus in UHV GIS Substations
}

\author{
Nian Guang Zhou' ${ }^{1}$, Qi Tang ${ }^{2, a}$, Xiao Wen $\mathrm{Wu}^{1}$ and Sheng $\mathrm{Hu}^{1}$ \\ ${ }^{1}$ State Grid Hunan Electric Power Corporation, Electric Power Research Institute, 410007 Changsha, China \\ ${ }^{2}$ Hunan Electric Experiment Research Institute, Environmental Protection Department, 410007 Changsha, China
}

\begin{abstract}
Investigation of audible noise characteristics of the main power apparatus in UHV GIS substations provides essential statistics for the noise prediction and control. Noise pressure level, spectrum and attenuation characteristics of the main transformers and high voltage (HV) reactors are measured and analyzed in this paper. The result shows that the main transformer and HV reactor have identical A-weighted equivalent sound pressure level. The medium- and low-frequency noises are the primary components in the spectral. More attention should be paid to the low-frequency bands in the noise control process. The noise of cooling fan has a large influence on that of the main transformer. Without the consideration of corona noise, the average A-weighted sound pressure level shows an overall decreasing trend with the increase of the propagation distance. Obvious interference phenomenon of the noises at 100 and $200 \mathrm{~Hz}$ exists in the noise propagation process.
\end{abstract}

\section{Introduction}

Audible noise has become the attention focus of the inhabitants around the substations. The research on the characteristic of UHV substation noise is particularly important and has practical significance in UHV power transmission project [1-3]. GIS equipment has numerous advantages, such as high reliability, less environmental impact and anti-electromagnetic interference. Besides, another significant advantage of GIS lies in that it has no audible corona noise, which reduces the noise emission level of substations [4].

The main noise of the UHV GIS substation comes from the transformer and $\mathrm{HV}$ reactor in operation. Compared with the conventional UHV substations, the noise characteristics of the GIS substation have some certain particularity. Hitherto, the noise characteristics of UHV substations are studied by some researchers [5]. However, few research reports are observed about the noise characteristics of UHV GIS substations.

In this paper, the audible noise characteristics of 1000 $\mathrm{kV}$ GIS substation in Anhui province of China has been investigated. The spectrum distribution and attenuation characteristics generated by transformers and reactors were analyzed in detail, which can provide reference to the acoustic environment impact prediction and assessment of different types of UHV substation.

\section{Description of tests}

The rated capacity of $1 \#$ transformer in the substation is $1000 / 1000 / 334$ MVA with the size of transformer is 11

\footnotetext{
${ }^{\mathrm{a}}$ Corresponding author: tqi081@163.com
}

$\mathrm{m} * 12 \mathrm{~m} * 5.5 \mathrm{~m}$ and the cooling mode of oil-immersed forced oil circulation air cooling(OFAF). Each phase of the transformer has 8 sets of cooling fans. There is a $17 \mathrm{~m}$ long and $9 \mathrm{~m}$ high firewall between two adjacent transformers. The distance from the firewall to the transformer is $8.5 \mathrm{~m}$ and $2.5 \mathrm{~m}$, respectively. In the test process, the active power of $1 \#$ transformer is -671.03 MW, meanwhile, the reactive power is 98.65 Mvar.

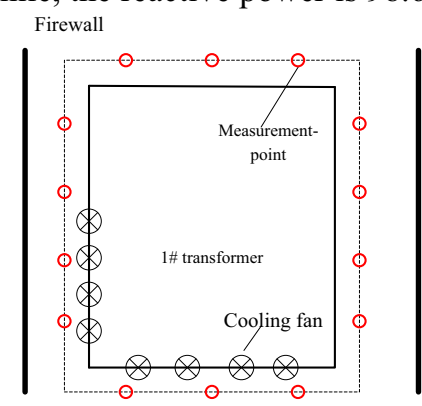

Figure 1. Measurement-point distribution around the transformer

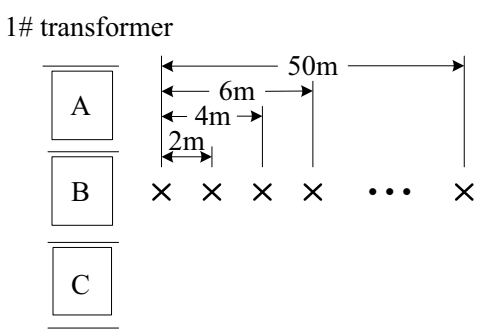

Figure 2. Measurement-point distribution of the transformer attenuation noise 
The measurement-point distribution around the transformer is shown in Figure 1. Figure 2 gives the measurement-point distribution of the transformer attenuation noise. The measurement section is located in the middle of the three phase transformers. The distance between the measurement points is $2 \mathrm{~m}$. Subjected to space constraints, 26 points are set in the measurement process.

Hu'An and Huai'Wu HV reactors are of the same type, of which the rated capacity is 240000 kvar. The size of reactor is $12 \mathrm{~m} * 10 \mathrm{~m} * 5.5 \mathrm{~m}$, and cooling mode adopts oil-immersed air cooling (ONAF). There is a $17 \mathrm{~m}$ long and $9 \mathrm{~m}$ high firewall between the two neighboring reactors. During the test, the active power of Hu'An line I is $1135.05 \mathrm{MW}$ and the reactive power is 247.78 Mvar. Meanwhile, the active power of Huai'Wu line II is 1670.34 MW and the reactive power -27.15 Mvar. The measurement-point distribution of the reactors and the reactor attenuation noise are identical to that of the transformer, as shown in Figure 3 and Figure 4, respectively.

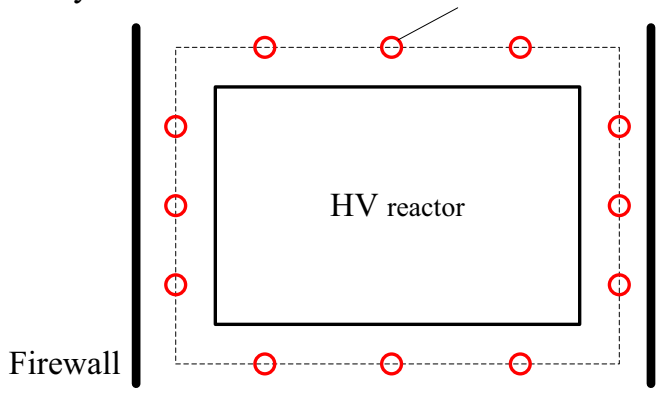

Figure 3. Measurement-point distribution around the reactor

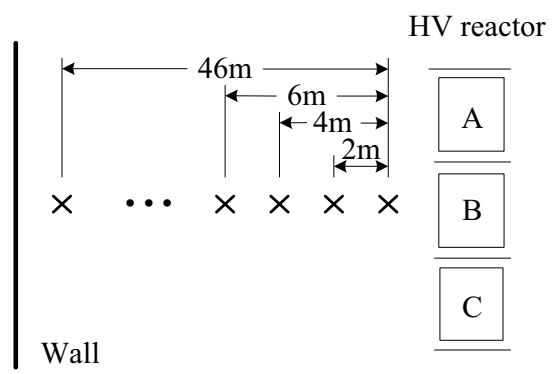

Figure 4. Measurement-point distribution of the reactor attenuation noise

\section{Results and discussion}

\subsection{Transformer Noise}

The test result of A-weighted equivalent sound pressure level $\left(L_{\text {Aeq }}\right)$ around each phase transformer is shown in Table 1. Because no noise sources are in the northern open-space of the main transformer and the only single cooling fan is shut down when the measurement is conducted, the minimum $L_{\text {Aeq }}$ of each transformer appears in the northern direction. The lowest sound pressure level of the three transformers is observed in Aphase, which is $67.2 \mathrm{~dB}(\mathrm{~A})$.
On the south side, two sets of cooling fans are running in the noise test process. Compared with the northern side, it is found that the operation of cooling fans makes the main transformer noise increases over $3 \mathrm{~dB}$ (A). Highest noise level appears in the west side of the transformer, which is $3.2 \sim 6.3 \mathrm{~dB}$ (A) higher than that of the northern side. This is attributed to the reason that the west side of the transformer is only $2.5 \mathrm{~m}$ away from the firewall, and the reflection phenomenon of the wall makes the noise of the side further enhanced to a large degree. On the other hand, there are 4 sets of cooling fans in the west side, one of which in operation. As the noise level of transformer is much affected by that of the wind turbine. Thus, in order to improve the calculation accuracy while predicting the noise distribution of UHV substations, the cooling fan of main transformers should be treated as independent sound source. A large calculation error will be caused if only take the transformer as area noise source with equivalent sound pressure level distribution. Moreover, in order to reduce the noise emission level, the low-noise fan should be considered as much as possible in the equipment selection process before the substation is put into service.

Table 1. Test results of average $L_{\text {Aeq }}$ around the main transformer

\begin{tabular}{ccccc}
\hline \multirow{2}{*}{ Phase } & \multicolumn{4}{c}{$\boldsymbol{L}_{\text {Aeq }} / \mathbf{d B}(\mathbf{A})$} \\
\cline { 2 - 5 } & East & South & West & North \\
\hline A & 73.3 & 73.4 & 73.5 & 67.2 \\
B & 72.8 & 73.9 & 74.3 & 71.0 \\
C & 70.6 & 72.1 & 72.9 & 69.7 \\
\hline
\end{tabular}

The 1/3-OCT spectrum distribution of the main transformer noise is shown in Figure 5. The spectrum distribution shows that A-weighted sound pressure level $\left(L_{\mathrm{A}}\right)$ of the main transformer in the range of $100 \sim 1600 \mathrm{~Hz}$ is relatively high. The maximum $L_{\mathrm{A}}$ is in the $315 \mathrm{~Hz}$ band, which belongs to the transformer body noise. The $L_{\mathrm{A}}$ decreases gradually as the frequency increases over 500 Hz. Influenced by the noise of cooling device, the middle- and high-frequency sound pressure level of the main transformer is relatively high.

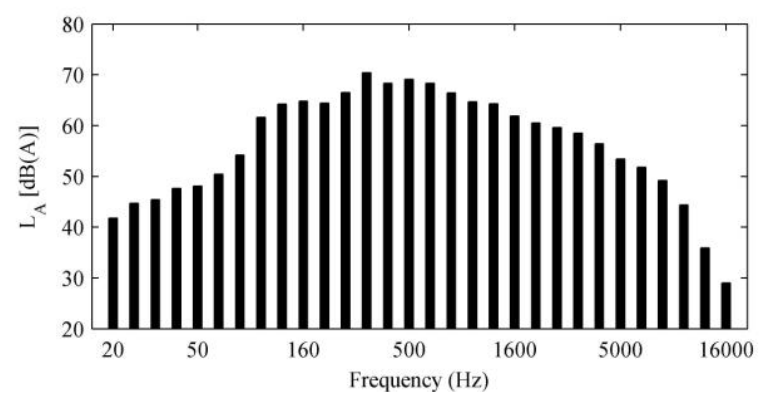

Figure 5. Spectrum distribution of the main transformer noise

The $L_{\text {Aeq }}$ was detected according to measurementpoints in Figure 2. The noise attenuation process of main transformer is shown in Figure 6. As the northern and southern side of the main transformer is relatively open, the sound pressure level is not affected by the corona noise generated on the power transmission structures 
such as the transmission lines, fittings and insulators compared with traditional UHV substations. Therefore, the noise attenuation processes of principal frequency components show the trend of decreasing gradually with the increase of the transmission distance. However, due to the phase difference of the transformer noises, interference phenomenon is observed in the noise transmission process. The noise level goes up and down to a certain degree depending on the distance other than a monotonous decline [5]. Thus, in order to improve the accuracy of noise prediction, the phase of main power transformers needs to be considered in the substation noise simulation model.

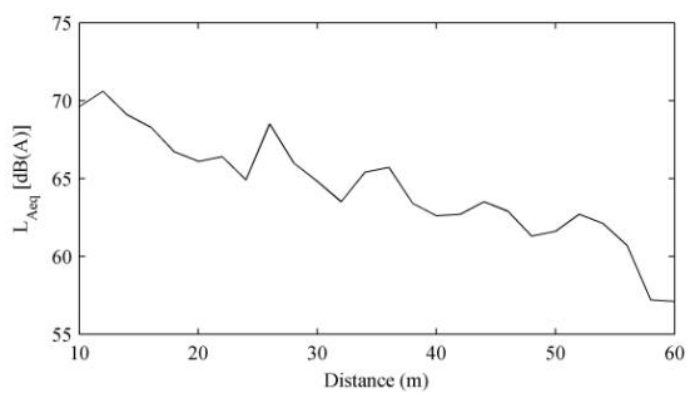

Figure 6. Noise attenuation process of the main transformer

The noise attenuation of the main transformer at various frequencies is shown in Figure 7. It is shown that in the noise propagation process, the fluctuations of the noise pressure level below $500 \mathrm{~Hz}$ are more obvious than that of 800 and $1000 \mathrm{~Hz}$. The noise fluctuations of 100 and $200 \mathrm{~Hz}$ are most significant. The largest fluctuation appears at the location of $50 \mathrm{~m}$ away from the main transformer. The above analysis shows that in the frequency range of $1000 \mathrm{~Hz}$ the noise propagation distance of 100 and $200 \mathrm{~Hz}$ frequency band in UHV substations is much further and the interference is the most serious. Thus, more attention should be paid to the low-frequency noises while controlling the transformer noises.

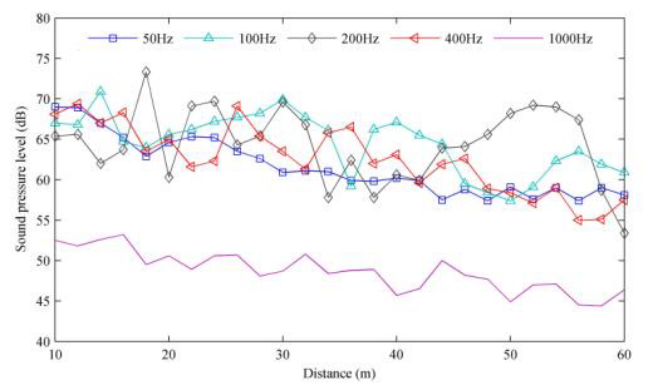

Figure 7. Noise attenuation of the main transformer at various frequencies

\section{$3.2 \mathrm{HV}$ reactor noise}

The result of $L_{\text {Aeq }}$ around the reactor of Hu'An line I and Huai'Wu line II is given in Table 2 and Table 3, respectively. It can be seen that the noise of $\mathrm{HV}$ reactors in UHV substation and that of transformers is at the same level. Reflected by the firewall, the noise level of reactor near the firewall is much higher. In the two reactor groups, the highest $L_{\mathrm{Aeq}}(78.2 \mathrm{~dB}(\mathrm{~A}))$ appears in the
phase-C reactor of Huai'Wu line II, while the minimum $L_{\text {Aeq }}(70.1 \mathrm{~dB}(\mathrm{~A}))$ appeared in the phase-A reactor of $\mathrm{Hu}$ 'An line I.

Table 2. Test results of average $L_{\mathrm{Aeq}}$ around the reactor of $\mathrm{Hu}$ 'An line I

\begin{tabular}{ccccc}
\hline \multirow{2}{*}{ Phase } & \multicolumn{4}{c}{$\boldsymbol{L}_{\text {Aeq }} / \mathbf{d B}(\mathbf{A})$} \\
\cline { 2 - 5 } & East & South & West & North \\
\hline A & 74.2 & 70.3 & 72.6 & 70.1 \\
B & 73.4 & 72.9 & 73.8 & 75.8 \\
C & 75.3 & 72.1 & 74.3 & 73.0 \\
\hline
\end{tabular}

Table 3. Test results of average $L_{\mathrm{Aeq}}$ around the reactor of Huai'Wu line II

\begin{tabular}{ccccc}
\hline \multirow{2}{*}{ Phase } & \multicolumn{4}{c}{$\boldsymbol{L}_{\text {Aeq }} / \mathbf{d B}(\mathbf{A})$} \\
\cline { 2 - 5 } & East & South & West & North \\
\hline A & 71.6 & 75.0 & 71.2 & 72.2 \\
B & 73.2 & 71.8 & 71.0 & 73.0 \\
C & 78.2 & 74.2 & 72.5 & 76.5 \\
\hline
\end{tabular}

The spectrum distribution of the $\mathrm{HV}$ reactor noise is shown in Figure 8 and Figure 9, respectively. Different from the frequency distribution characteristics of UHV transformer noise, the high noise level of the reactor mainly concentrates in the $100 \mathrm{~Hz}$ band, which is mainly caused by the expansion of core lamination and electromagnetic force of the winding. Therefore, the body noise should be considered in priority when controlling the HV reactor noise.

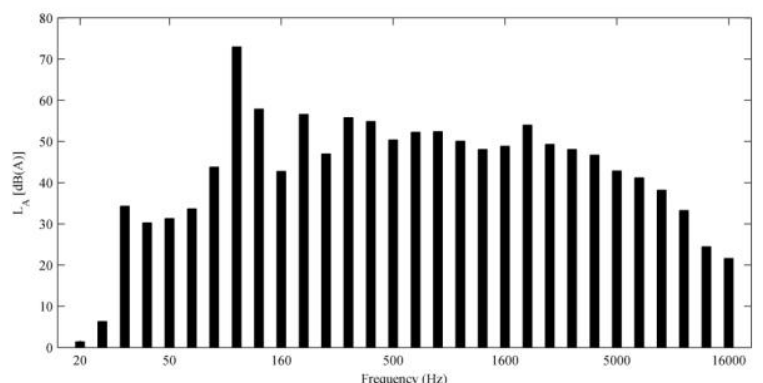

Figure 8. Spectrum distribution of the $\mathrm{HV}$ reactor (Hu'An line I)

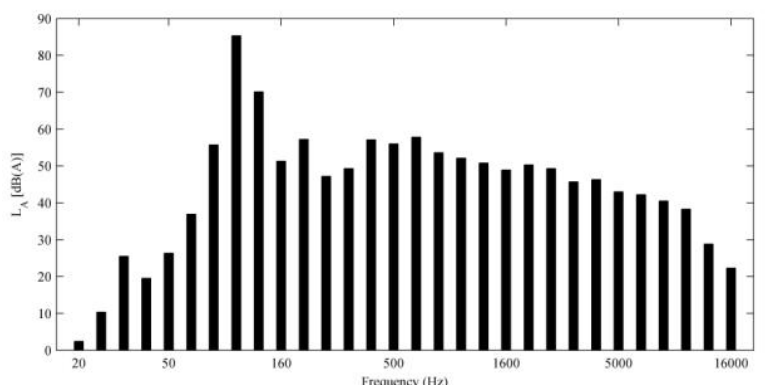

Figure 9. Spectrum distribution of the $\mathrm{HV}$ reactor (Huai'Wu line II)

The noise attenuation process of the two sets of HV reactors is shown in Figure 10. It can be seen that the noise attenuation process of the $\mathrm{HV}$ reactor is similar to the process of transformer. There is obvious interference in the propagation process of $\mathrm{HV}$ reactor noise [9]. Except that the sound pressure level near the wall increases due to reflection, the sound pressure level of 
Hu'An line I within the range of $30 \mathrm{~m}$ shows a monotonically decreasing trend. Compared with the reactor of Hu'An line I, the noise fluctuation of Huai' Wu line II is more significant. The noise pressure level, which locates at 14 and $36 \mathrm{~m}$ away from the $\mathrm{HV}$ reactor, is close to that of the measurement-point near the HV reactor. The overall trend of sound pressure level within the scope of $50 \mathrm{~m}$ doesn't decrease gradually. The reason for this phenomenon may be that the west and south of Huai'Wu line II are surrounded by high wall, and the distance is pretty close. The distance between the reactor and the west wall is only $30.5 \mathrm{~m}$. Owing to the reflection of the wall and the interference between acoustic waves, the noise on that side maintains a high level and the attenuation process is relatively slow.

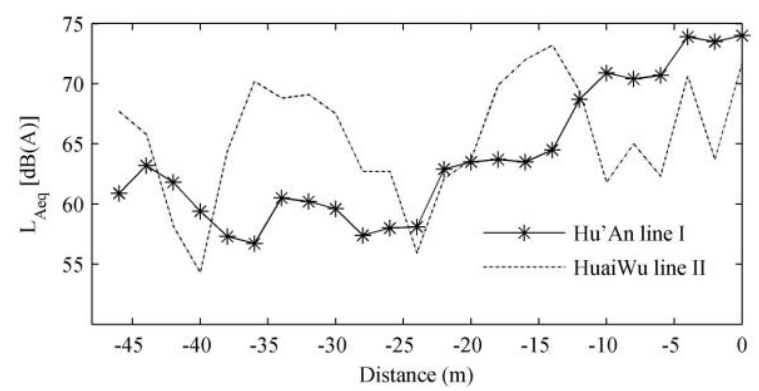

Figure 10. Noise attenuation of the HV reactors

In general, the location of $\mathrm{HV}$ reactors in UHV substation is near the wall. Because of the low frequency and the long propagation distance, the wall reflection and the interference between acoustic waves usually lead to the noise emission level in the region can't meet the specified value in the standards. Hence, in the planning and designing stage of UHV substations, the position of noise-sensitive point and the layout of HV reactor should be take into account.

The noise attenuation processes of HV reactors in different frequency band are shown in Figure 11 and Figure 12, respectively. It is shown that, the fluctuations of sound pressure levels at 100 and $200 \mathrm{~Hz}$ is most obvious in the process of sound transmission. Moreover, the noise of $1000 \mathrm{~Hz}$ decreases with the increase of the propagation distance. It can be concluded that the noise attenuation process of $\mathrm{HV}$ reactors is similar to that of transformers, and the noise interference at 100 and $200 \mathrm{~Hz}$ is most obvious. In order to realize accurate prediction of the sound field distribution of the reactor, phase information of each noise source needs consideration in the calculation.

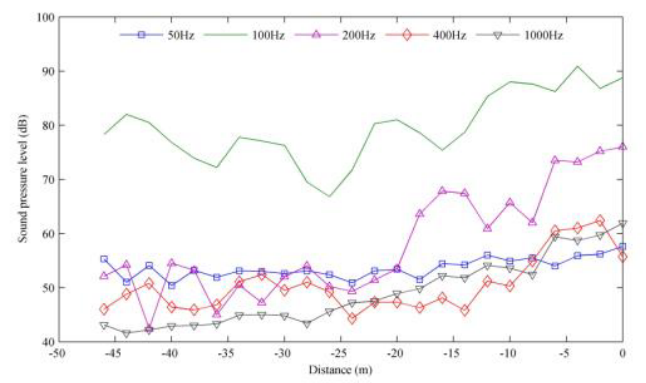

Figure 11. Noise attenuation of the HV reactor at various frequencies (Hu'An line I)

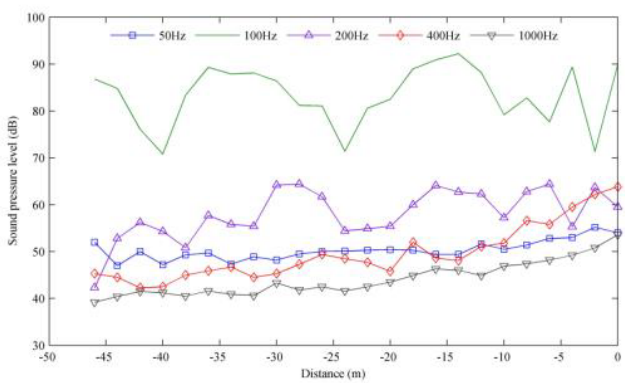

Figure 12. Noise attenuation of the HV reactor at various frequencies (Huai'Wu line II)

\section{Conclusions}

In this paper, the noise pressure level, spectrum and attenuation characteristics of Wuhu UHV GIS substation are measured and analyzed. The following conclusions can be drawn:

(a) The noise of the main transformers and $\mathrm{HV}$ reactors in UHV GIS substations are mainly in middleand low-frequency. However, the noise level of the HV reactor is relatively higher than that of main transformer. The cooling fan has a large effect on the noise of main transformer.

(b) Since the noise source of the UHV GIS substation does not include the corona noise generated on the power transmission structures, the average $L_{\text {Aeq }}$ shows an overall decreasing trend with the increase of the propagation distance.

(c) Interference phenomenon exists in the noise propagation process of the main transformers and $\mathrm{HV}$ reactors, and the interference of 100 and $200 \mathrm{~Hz}$ is most obvious. Layout optimization of the HV reactors could reduce the noise emission level at the substation boundary.

\section{References}

1. Y. Chen, H. Xie and Y. Zhang. High Voltage Engineering, 38, 2189-2194 (2012)

2. G. Di, X. Zhou and X. Chen. Applied Acoustics, 91, 40-46 (2015)

3. J. Zhou, L. Li and Y. Du. Electric Power, 42, 75-78 (2009)

4. R. Nagarsheth, S. Singh. International Journal of Electrical Power \& Energy Systems, 55, 481-485 (2014)

5. B. Zhou, C. Pei and Y. Ni. High Voltage Engineering, Vol. 39, 1447-1453 (2013)

6. H. Ma, J. He and Q. Chen. High Voltage Engineering, 34, 1599-1604 (2008)

7. J. Mo, X. Zhang and J. Yuan. Advanced Technology of Electrical Engineering and Energy, 34, 76-80 (2015)

8. Y. Yu, B. Chen. Transformer, 44, 23-44 (2007)

9. Y. Ni, B.Zhou and C.Pei. High Voltage Engineering, 40, 3926-3932 (2014) 